\title{
Class actions: Neuer Zugang zum Verwaltungsrecht?
}

\author{
Dr. Torben Ellerbrok
}

\begin{abstract}
Der Beitrag geht der Frage nach, ob ein kollektives Rechtsschutzverfahren, bei dem - nach Art der US-amerikanischen class action - für gleichartig Betroffene in einem einzigen Verfahren ein einheitliches Urteil gefällt wird, den Zugang zum deutschen Verwaltungsrecht verbessern könnte. Dazu werden die beiden zentralen Wirkungen, zum einen die Verbesserung der Prozessökonomie, zum anderen die Effektuierung der Rechtsdurchsetzung, für den Verwaltungsprozess näher betrachtet und mögliche Anwendungsbereiche im Verwaltungsrecht aufgezeigt. Anschließend wird untersucht, inwieweit sich ein kollektiver Rechtsschutz in die Konzeption der deutschen Verwaltungskontrolle, die verfassungsrechtlichen Vorgaben und die derzeitige, auf einen individuellen Rechtsschutz ausgerichtete Ausgestaltung des Verwaltungsprozessrechts einfügen würde. Rechtspolitische Erwägungen schließen - unter Berücksichtigung bereits bestehender Vorkehrungen des Verwaltungsprozessrechts für Massenverfahren den Beitrag ab.
\end{abstract}

\section{Einfübrung}

Versteht man die Frage nach dem Zugang zu materiellem Recht als Frage nach der Möglichkeit seiner Verwirklichung und Durchsetzung, so entscheidet darüber maßgeblich die Ausgestaltung des Prozessrechts. ${ }^{1}$ Für den Zugang zum Verwaltungsrecht wird insofern die Verwaltungsgerichtsordnung (VwGO) relevant, ${ }^{2}$ mit der das Verwaltungsprozessrecht 1960 bundeseinheitlich kodifiziert wurde und die seitdem nur punktuell Ergän-

* Herzlicher Dank gilt dem Team der JTÖR für die hervorragende Organisation der Tagung und allen Diskutantinnen und Diskutanten sowie Dr. Alexander Brade und Dr. Konstantin Chatziathanasiou für wertvolle Anregungen.

1 Auch Jochum, Verwaltungsverfahrensrecht und Verwaltungsprozeßrecht, 2004, S. 98.

2 Finanz- und sozialgerichtliches Verfahren bleiben hier außer Betracht, die Erwägungen lassen sich grds. übertragen. 
zungen und Erweiterungen erfahren hat. ${ }^{3}$ Die Verwaltungstätigkeit ist derweil in stetem Wandel begriffen. In den vergangenen Jahrzehnten haben dabei unter anderem Massenbetroffenheiten durch Verwaltungshandeln, also die aus rechtlicher Sicht identische Betroffenheit einer Vielzahl von Personen, erheblich zugenommen: Erstens hat sich die deutsche Verwaltung von einer vorrangig eingreifenden zu einer stärker leistenden, planenden, regulierenden und informierenden Verwaltung mit hoher Breitenwirkung fortentwickelt. Zweitens wurde die rechtliche Determination des Verwaltungshandelns in bestimmten Rechtsgebieten, etwa dem Immissionsschutzrecht, erheblich ausgeweitet, wodurch rechtliche Ansprüche für einen größeren Personenkreis überhaupt erst entstanden sind. Schließlich drittens befördern eine Zentralisierung des Verwaltungsvollzugs durch die Schaffung von Sonderbehörden und eine zunehmend automatisierte Arbeitsweise der Verwaltung inhaltsgleiche Verwaltungsentscheidungen für eine Vielzahl von Bürgerinnen und Bürgern ${ }^{4}$. Ohne den besonderen Bedarf für die rechtliche Stabilität von Prozessordnungen in Abrede zu stellen, rechtfertigt dies, wieder ${ }^{5}$ verstärkt darüber nachzudenken, ob und inwieweit der deutsche Verwaltungsrechtsschutz für Massenbetroffenheiten hinreichend eingerichtet ist oder Verbesserungsmöglichkeiten für einen effektiven Zugang zum Verwaltungsrecht bestehen. Dieser Beitrag widmet sich dabei einem weitreichenden Innovationsansatz: Class actions sind eine „besondere Klageart“ ${ }^{\text {" }}$, bei der gleichartige Ansprüche im Kollektiv verfolgt werden (II.). Beleuchtet wird, ob und inwieweit die Einführung eines solchen kollektiven Rechtsschutzes in das deutsche Verwaltungsprozessrecht als Ergänzung zum bestehenden individuellen Rechtsschutz Potential hat, durch eine prozessökonomische Wirkung und eine Rechtsdurchsetzungswirkung den Zugang zum Verwaltungsrecht zu verbessern (III.). Darauf aufbauend wird der Frage nachgegangen, ob class actions eine systemkongruente (IV.) und rechtspolitisch erstrebenswerte (V.) Ergänzung des bestehenden Verwaltungsprozessrechts sein könnten.

3 Zusammenfassend Held, in: Sommermann/Schaffarzik, Handbuch der Geschichte der Verwaltungsgerichtsbarkeit in Deutschland und Europa, Bd. 1, 2019, S. 989 (991 ff.).

4 Aus Platzgründen wird im Folgenden auf die Verwendung aller Sprachformen verzichtet. Sämtliche Bezeichnungen gelten stets für alle Geschlechter.

5 Vgl. schon Laubinger, Gutachten über eine künftige gesetzliche Regelung für Massenverfahren im Verwaltungsverfahrensrecht und im Verfahrensrecht für die Verwaltungsgerichte, 1975.

6 Ebbing, ZVglRWiss 103 (2004), 31 (32). 


\section{Class action: Begriff und US-amerikanische Ausgestaltung}

Class actions oder "Gruppenklagen" ${ }^{7}$ ermöglichen die Erhebung einer Klage durch einen Repräsentanten ${ }^{8}$, um für sich und weitere Personen (class oder Gruppe) identische oder jedenfalls gleichartige Fragen rechtlicher und/oder tatsächlicher Art in einem einzigen gerichtlichen Verfahren rechtsverbindlich zu entscheiden. ${ }^{9}$ International werden class actions heute vor allem als US-amerikanische Form des Rechtsschutzes wahrgenommen. ${ }^{10}$ Nach dem - hier exemplarisch herangezogenen - Bundesverfahrensrecht der USA kann jede Person Klage ${ }^{11}$ als class action erheben. Sie wird vom Gericht als class action zugelassen, wenn erstens eine Tatsachenoder Rechtsfrage eine Mehrzahl von Personen betrifft, zweitens der vom Kläger geltend gemachte Anspruch typisch für die Angehörigen dieser Gruppe ist, drittens der Kläger Gewähr bietet, als Repräsentant die Interessen der gesamten Gruppe zu vertreten, und schließlich viertens die Zahl der Betroffenen so groß ist, dass ein Prozess im Wege der Klageverbindung nicht durchführbar wäre (Rule 23 [a] der Federal Rules of Civil Procedure). ${ }^{12}$ Zumeist $^{13}$ dient die class action dazu, in einem einheitlichen Verfahren individuelle und voneinander unabhängige Ansprüche einzuklagen, bei denen identische Rechts- und/oder Tatsachenfragen die Einzelfallspezifika überwiegen (Rule 23 [b] [3]). Dann muss das Gericht alle Gruppenangehörigen informieren bzw., sofern diese nicht im Einzelnen bekannt sind, sich mittels "the best notice that is practicable“ an die Angehörigen der class richten (Rule 23 [c] [2] [B]). So werden diese insbesondere auf ihr Austrittsrecht (sog. opt-out-Modell) hingewiesen. Wer davon keinen Gebrauch macht, wird von der Rechtskraft der gerichtlichen Entscheidung erfasst (Rule 23 [c] [3] [B]). Mit dieser kurzen Einführung kann und soll

7 Teilweise wird - ebenfalls synonym - von Sammelklagen gesprochen, z. B. Hohl, Die US-amerikanische Sammelklage im Wandel, 2008.

8 Krit. zu diesem Begriff Lange, Das begrenzte Gruppenverfahren, 2011, S. 86, 122.

9 Bisweilen wird ergänzt, dass die Mitglieder der Gruppe nicht „Parteien des Verfahrens" sind, so z. B. Fiedler, Class Actions zur Durchsetzung des europäischen Kartellrechts, 2010, S. 9.

10 Cox, Arizona Law Review 39 (1997), 497 (497): „Few things are as American as the class action".

11 Die Konstellation, in der eine Person stellvertretend auf Beklagtenseite steht, bleibt hier außer Betracht.

12 Ausf. Beuchler, Class Actions und Securities Class Actions in den Vereinigten Staaten von Amerika, 2008, S. $81 \mathrm{ff}$.

13 Krit. und zur Bedeutung weiterer Varianten Carroll, Duke Law Journal 65 (2016), $843 \mathrm{ff}$. 
es hier sein Bewenden haben, da es im Folgenden nicht um die Frage nach einer Übertragung des US-amerikanischen Modells in Reinform, sondern um eine rechtsvergleichend informierte Einführung von Kollektivklagemöglichkeiten geht.

\section{Wirkungen und Potentiale einer class action}

In Deutschland wurde die Einführung kollektiver Rechtsschutzinstrumente vielfach diskutiert, ${ }^{14}$ allerdings - obwohl sich class actions im Rechtskreis des Common Law auch gegen den Staat und seine Untergliederungen richten können ${ }^{15}$ - nicht für das Verwaltungs- ${ }^{16}$ sondern nahezu ausschließlich für das Zivilprozessrecht. ${ }^{17}$ Der deutsche Gesetzgeber agierte bisher auch im Hinblick auf den Zivilprozess zurückhaltend, hat aber zuletzt durch das Kapitalanleger-Musterverfahrensgesetz (KapMuG) und die Musterfeststellungsklage ( $\mathbb{S} 606 \mathrm{ff}$. ZPO) Varianten der class action eingeführt; unionsrechtliche Regelungen lassen hier Erweiterungen erwarten. ${ }^{18}$ Schon die vielfache Durchbrechung der in Art.95 S. 1 GG angelegten Trennung der Gerichtsbarkeiten durch abdrängende Sonderzuweisungen und die entsprechende Anwendung zivilprozessualer Regelungen im nur lückenhaft normierten Verwaltungsprozess (vgl. $\mathbb{S} 54$ Abs. 1, 57 Abs. 2, 64,

14 Bruns, NJW 2018, 2753 (2753): „rechtspolitische[r] Dauerbrenner“; Schneider, BB 2018, 1986 (1986): „Evergreen in Rechtswissenschaft und Rechtspolitik“.

15 Dies gilt jedenfalls für die USA, Kanada und Australien, ausf. mit Bsp. Mulheron, Class Actions and Government, 2020, S. $262 \mathrm{ff}$.

16 In diese Richtung, allerdings mit rechtspolitischen Ausführungen nur zur Verbandsklage Gluding, Kollektiver und überindividueller Rechtsschutz im Zivilund Verwaltungsprozessrecht, 2020, S. 450 ff. Laubinger, Gutachten (Fn. 5), S. 18 ff., konstatierte: „Die in einer jahrhundertealten Rechtstradition wurzelnde Class Action dürfte sich für eine Übertragung in das deutsche Recht nicht eignen".

17 Frühzeitig Gottwald, ZZP 91 (1978), 1 ff.; Koch, Kollektiver Rechtsschutz im Zivilrecht, 1976; Mertens, ZHR 139 (1975), 438 ff.; zuletzt Fries, Verbraucherrechtsdurchsetzung, 2016, S. 170 ff.; Meller-Hannich, Verhandlungen des 72. Deutschen Juristentages, Bd. I, 2018, A 1 ff.; zu Überlegungen in Österreich Klever/Schwamberger, GVRZ 2020, 4 Rn. 32 ff. m.w.N.

18 Zuletzt Richtlinie (EU) 2020/1828 des Europäischen Parlaments und des Rates v. 25.11.2020 über Verbandsklagen zum Schutz der Kollektivinteressen der Verbraucher und zur Aufhebung der Richtlinie 2009/22/EG, ABl L 409/1. 
$173 \mathrm{VwGO})^{19}$ zeigen aber, dass verwaltungsrechtliche Fragen nicht in allen Bereichen und Belangen nach einer prozessualen Sonderbehandlung verlangen. Vor diesem Hintergrund stellt sich die Frage, ob und inwieweit kollektive Rechtsschutzverfahren (auch) im deutschen Verwaltungsrecht einen Mehrwert entfalten können.

\section{Massenbetroffenheit im Verwaltungsrecht}

Class actions ergeben nur Sinn, wenn mehr als eine Person von einem Sachverhalt betroffen ist, der in den aus rechtlicher Sicht relevanten Bestandteilen entweder vollständig oder zumindest im Hinblick auf näher festzulegende Kriterien identisch ist, und sich daraus Rechtsansprüche ergeben. Die Betroffenheit kann dabei entweder aus derselben Ursache (echte Massenbetroffenheit) oder aus mehreren, in gewisser Hinsicht gleichen Ursachen resultieren (unechte Massenbetroffenheit).

Verwaltungshandeln erfolgt vielfach individuell und einzelfallbezogen. Gleichwohl ergeben sich hier mannigfaltige Konstellationen, die für class actions geeignet sind. Denn Verwaltungstätigkeit zeichnet sich einerseits durch die Genehmigung (teilweise auch Umsetzung) von Großvorhaben (insb. Infrastrukturprojekten wie Autobahnen, Bahnhöfen, Flughäfen, Kraftwerken) aus, die eine hohe Breitenwirkung aufweisen und damit eine echte Massenbetroffenheit generieren. Andererseits hat die öffentliche Verwaltung eine Art natürliche Monopolstellung inne. Daraus resultiert eine Vielzahl von Massenverfahren, etwa bei der Leistungsvergabe in der Sozialverwaltung oder der Gebührenerhebung, bei denen Personen in rechtlich identischer Weise betroffen sind. Hier befördern - neben der bereits in der Einleitung benannten Automatisierung - der Vorbehalt des Gesetzes und der Gleichbehandlungsgrundsatz (Art. 3 Abs. 1 GG) die Konvergenz von Verwaltungsentscheidungen.

\section{Prozessökonomische Wirkung}

Bei einer individuell mittleren oder starken Massenbetroffenheit kann class actions prozessökonomische Wirkung zukommen.

19 Ausf. Nolte, Die Eigenart des verwaltungsgerichtlichen Rechtsschutzes, 2015, S. $203 \mathrm{ff}$. Der Gesetzgeber hat durch $\$ 173$ S. 1 Hs. 2 VwGO eine Anwendung der $\$ \$ 606 \mathrm{ff}$. ZPO im Verwaltungsprozess ausgeschlossen. 


\section{a) Prozessökonomie}

Prozessökonomie - ungeschriebener Grundsatz des deutschen Prozessrechts ${ }^{20}$ - meint die Feststellung streitentscheidenden Rechts unter Inanspruchnahme möglichst geringer gerichtlicher Ressourcen. ${ }^{21}$ Darauf können class actions einen positiven Effekt haben, indem sie durch die Bündelung des Verfahrensstoffes eine „ressourcenintensive Mehrfachbefassung“22 vermeiden: ${ }^{23}$ In einem einheitlichen Verfahren für eine Vielzahl identischer Betroffenheiten müssen Sachverhalte nur einmal ermittelt, Beweiserhebungen nur einmal durchgeführt, Sachverständige nur einmal bestellt ${ }^{24}$ werden. Eine class action eröffnet zudem Raum für organisatorische Vorteile. So ermöglicht die eindeutige Zuweisung einer Repräsentationsbefugnis, die Herausforderungen eines Verfahrens mit einer hohen Zahl von Beteiligten (räumliche Kapazitäten, Ladung etc.) erheblich zu reduzieren. Die prozessökonomische Wirkung der class action tritt freilich nur ein, wenn auch im Wege individuellen Rechtsschutzes eine gewisse Mindestanzahl von Gerichtsverfahren angestrengt worden wäre. Dies setzt voraus, dass die individuelle Betroffenheit ein gewisses Maß übersteigt (individuell mittlere oder starke Massenbetroffenheit ${ }^{25}$ ). Zudem generieren class actions zusätzlichen Aufwand für die Gerichte, der die prozessökonomische Wirkung reduziert: Die Gruppe muss definiert werden, ggf. muss die Beweisaufnahme vielschichtiger und umfassender erfolgen und müssen Entscheidungen öffentlich bekanntgemacht werden.

20 Für das Verwaltungsprozessrecht BVerwGE 116, 188 (197); 161, 76 Rn. 11; BVerwG, NVwZ 2018, 1229 (1230); Menger, System des verwaltungsgerichtlichen Rechtsschutzes, 1954, S. 246; Ortloff, in: Schoch/Schneider, VwGO, $\mathbb{1} 104$ Rn. 19 (Stand: 2010).

21 Vgl. Hyckel, Prozessökonomie, 2020, S. 323; ders., GVRZ 2020, 5 Rn. 76.

22 Fries, Verbraucherrechtsdurchsetzung (Fn. 17), S. 177. Heese, JZ 2019, 429 (431) meint, bei Massenschäden führe die Vielzahl von Individualprozessen zu einer „maßlosen Verschwendung staatlicher Ressourcen“.

23 Auch Ebbing, ZVglRWiss 103 (2004), 31 (45).

24 Darauf verweist auch - betreffend das KapMuG - der Entwurfsgesetzgeber: BTDrs. 15/5091, S. 17.

25 In der zivilrechtlichen Diskussion wird analog zwischen (qualifizierten) „Massenschäden“ (Amrhein, Die Musterfeststellungsklage, 2020, S. 32 f.; Dürr-Auster, Die Qualifikation als Gruppen- oder Verbandskläger im kollektiven Rechtsschutz, 2017, S. 8 f.) bzw. „Großschäden“ (Ebbing, ZVglRWiss 103 [2004], 31 [37 f.]) und "Streuschäden“ (Towfigh/Chatziathanasiou, in: Schulte-Nölke u.a., Neue Wege zur Durchsetzung des Verbraucherrechts, 2017, S. 93 [99]) unterschieden. 


\section{b) Anwendungspotentiale im deutschen Verwaltungsrecht}

Die Bereiche einer individuell mittleren oder starken Massenbetroffenheit, in denen Verwaltungsgerichte aufgrund gleichgearteter und -gerichteter Klagen derzeit wiederholt über dieselbe Tatsachen- und/oder Rechtsfrage zu entscheiden haben, sind im Verwaltungsrecht vielgestaltig und lassen sich kaum auf spezifische Bereiche eingrenzen. Exemplarische Konstellationen einer echten Massenbetroffenheit sind die Genehmigung eines Bauvorhabens, gegen die allen Grundstückseigentümern im selben Plangebiet auf Grundlage eines Gebietserhaltungsanspruchs ein Anfechtungsrecht zusteht, oder behördliche Warnungen vor dem Verzehr eines Lebensmittels, wobei - mangels verifizierter wissenschaftlicher Grundlage - sämtlichen Herstellern gegen die Behörde ein Unterlassungsanspruch zukommt. Typische Konstellationen einer unechten, individuell mittleren oder starken Massenbetroffenheit im Verwaltungsrecht sind etwa die Erhebung von Straßenausbaubeiträgen von Grundstückseigentümern oder die Versagung der Zulassung zahlreicher Bewerber zu einem Studiengang aus Kapazitätsgründen. ${ }^{26}$

\section{Rechtsdurchsetzungswirkung}

Bei einer individuell geringen Betroffenheit kann class actions zudem eine Rechtsdurchsetzungswirkung zukommen.

\section{a) Klageerhebung als Kosten-Nutzen-Abwägung}

$\mathrm{Ob}$ und inwieweit ein Bürger einen Rechtsanspruch im Klageweg durchsetzt, lässt sich aus einer ökonomisch informierten Perspektive als KostenNutzen-Abwägung eines eigennutzmaximierenden homo oeconomicus darstellen: Rational ist die Klageerhebung, wenn der Erwartungsnutzen eines Prozesses die erwarteten Kosten übersteigt. ${ }^{27}$ Der Nutzen eines Prozesses ergibt sich dabei als Produkt der Erfolgswahrscheinlichkeit und des aus

26 Für Verteilungsentscheidungen wie die Studienplatzvergabe weisen class actions durch das einheitliche Verfahren und die einheitliche Entscheidung zusätzliche Vorteile auf.

27 Vgl. zu dieser Grundannahme Poelzig, Normdurchsetzung durch Privatrecht, 2012, S. 379; van den Bergh/Keske, in: Casper/Janssen/Pohlmann/Schulze, Auf dem Weg zu einer europäischen Sammelklage?, 2009, S. 17 (20). 
einem Prozesserfolg resultierenden Mehrwerts. Dieser liegt bei einem Unterlassungsanspruch im Ausbleiben künftiger Beeinträchtigungen, bei einem Geldzahlungsanspruch in der Vermögensmehrung, bei einem Feststellungsanspruch mitunter auch nur in der „Genugtuung“ oder Klarstellung. Dem stehen die (subjektiven Erwartungs-)Kosten einer prozessualen Geltendmachung gegenüber. Die Gerichtskosten, mitsamt zusätzlicher Kosten für eine Beweiserhebung, und die Rechtsanwaltsgebühren (aller Verfahrensbeteiligter) müssen dabei im Hinblick auf die spezifisch vorgesehene Kostentragungspflicht (im Verwaltungsprozessrecht gilt der Unterliegensgrundsatz $[\mathbb{S} 154 \text { Abs. } 1 \mathrm{VwGO}]^{28}$ und deren Eintrittswahrscheinlichkeit ${ }^{29}$ in die Abwägung eingestellt werden. Weitere, nicht auf einen Prozessunterlegenen überwälzbare, vor allem aber auch von Rechtsschutzversicherungen allenfalls eingeschränkt erstattete Kosten resultieren aus dem Aufwand zur Ermittlung von Tatsachen und Aufbereitung von Beweismitteln, ${ }^{30}$ um das Bestehen und die Durchsetzbarkeit eines Anspruchs zu prüfen. Der zeitliche Aufwand für eine prozessuale Durchsetzung erzeugt Opportunitätskosten. ${ }^{31}$ Schließlich sind auch die notwendige Überwindung einer psychologischen Hemmschwelle ${ }^{32}$ sowie mögliche negative Reaktionen des Beklagten ${ }^{33}$ als Kosten zu berücksichtigen.

Ergibt sich aus alledem ein negativer Nettonutzen (Kosten $>$ Nutzen), besteht ein „rationales Desinteresse“ 34 an der gerichtlichen Rechtsverfolgung. Dies führt zu einer „rationalen Apathie“"35; eine Rechtsdurchsetzung bleibt in der Regel aus, da im Verwaltungsrecht auch eine außergerichtliche Einigung zumeist ausscheidet. Die exakte Bestimmung einer Rationali-

$28 \mathrm{Zu}$ berücksichtigen ist die gem. $\$ 162 \mathrm{VwGO}$ begrenzte Erstattungsfähigkeit.

29 Eine Ungewissheit über den Ausgang ist als „treibende Kraft“ (Luhmann, Legitimation durch Verfahren, 3. Aufl. 1978, S. 116) einem Gerichtsverfahren immanent.

30 Auch Haß, Die Gruppenklage, 1996, S. 30.

31 Auch Schwalbe, in: Schmidt-Kessel/Strünck/Kramme, Im Namen der Verbraucher?, 2015, S. 23 (25 f.).

32 Nagy, Columbia Journal of European Law 19 (2013), 469 (474); auch BT-Drs. 19/243, S. 16: „Ungewohntes und Unerwünschtes“.

33 Auch Poelzig, Normdurchsetzung (Fn. 27), S.381. Anonymität und rechtliche Prägung des Verhältnisses zwischen Bürger und Verwaltung lassen erwarten, dass die Wahrscheinlichkeit für emotional-individuelle Konsequenzen geringer ist.

34 Schäfer/Ott, Lehrbuch der ökonomischen Analyse des Zivilrechts, 6. Aufl. 2020, S. 459; ferner Ebbing, ZVglRWiss 103 (2004), 31 (45); Schwalbe, Verbraucher (Fn. 31), S. 23 (29).

35 Dür-Auster, Qualifikation (Fn. 25), S. 13 m.w.N.; Poelzig, Normdurchsetzung (Fn. 27), S. 40, 382; Wagner, ZEuP 2008, 6 (15). 
tätsschwelle ist dabei ausgeschlossen, ${ }^{36}$ denn Nutzen und Kosten sind maßgeblich von individuellen Faktoren (Vermögen, Intellekt, Risikoaversion, Stressresistenz, sonstige zeitliche Beanspruchung etc.) abhängig. Da aber durch eine Klageerhebung gewisse Fix- und Mindestkosten entstehen, ist jedenfalls die Wahrscheinlichkeit für einen negativen Nettonutzen umso höher, je geringer die individuelle Betroffenheit ist.

\section{b) Stärkung durch class actions}

Sofern die Ansprüche nicht rivalisieren, hat eine Rechtsverfolgung im Wege einer class action keine Auswirkungen auf den durchsetzbaren Anspruch und damit grundsätzlich ${ }^{37}$ auch nicht auf die individuellen Nutzen eines Prozesses für einen Anspruchsberechtigten. Die veränderten Rahmenbedingungen prozessualer Geltendmachung können aber - für verwaltungsebenso wie für privatrechtliche Ansprüche - zu einer Reduzierung der Kosten führen: Durch die Bekanntmachung einer class action verringern sich regelmäßig ganz erheblich die Informationskosten für die Prüfung und Erkenntnis, ob überhaupt ein Rechtsverstoß vorliegt. Bei der gerichtlichen Durchsetzung treten durch eine Bündelung der Ansprüche Skaleneffekte ein, soweit Gerichtskosten und Rechtsanwaltsgebühren - wie nach derzeitiger Ausgestaltung - nicht proportional zu der Anzahl der Kläger steigen. Schließlich entsteht durch das initiierte Vorgehen als Kollektiv ein informationaler Einfluss ${ }^{38}$, der auch die Verhaltenserwartung für Gruppenangehörige ${ }^{39}$ beeinflussen kann und so die individuelle Hemmschwelle für eine Rechtsdurchsetzung senkt. Im Ergebnis führt dies zu einer Kostenreduzierung gegenüber der Individualklage, sofern zum einen die zusätzlichen Organisationskosten für das Auffinden und die Koordination der Gruppe hinreichend gering gehalten werden. Dafür bietet das Internet in Form einer leicht zugänglichen Bekanntmachung auf spezifischen Por-

36 Ebenso Poelzig, Normdurchsetzung (Fn. 27), S.41. Für die zivilprozessuale Rechtsdurchsetzung wird eine Grenze mit $150 €$ (Burckhardt, Auf dem Weg zu einer class action in Deutschland?, 2005, S. 122 ff., insb. S. 128) oder $200 €$ (Fiedler, Class Actions [Fn. 9], S. 40) beziffert.

37 Denkbar ist, dass sich die Erfolgswahrscheinlichkeit aufgrund eines gesteigerten Einsatzes im Prozess erhöht oder aufgrund größerer Zurückhaltung der Gerichte reduziert.

38 Dazu schon Deutsch/Gerard, Journal of Abnormal and Social Psychology 51 (1955), $629 \mathrm{ff}$.

39 Weiterführend Brown/Pehrson, Group Processes, 3. Aufl. 2020, S. $51 \mathrm{ff}$.; Nijstad/ van Knippenberg, in: Jonas/Stroebe/Hewstone, Sozialpsychologie, 2014, Rn. 12.4.2. 
talen und einer jederzeit durchführbaren Online-Anspruchsprüfung durch automatische Datenverarbeitung entscheidende Möglichkeiten. Zum anderen sind hinreichende Anreize notwendig, um die Rechtsdurchsetzung nicht durch Kollektivhandlungsprobleme wie ein Trittbrettfahrerverhalten zu gefährden. Wird im Ergebnis durch eine Kostensenkung in kollektiven Rechtsschutzverfahren die prozessuale Durchsetzung eines Anspruchs bei einer individuell geringen Betroffenheit attraktiv(er), ${ }^{40}$ werden ,autonomiebedingte Kontrolldefizite" 41 überwunden. Die class action wird zum Rechtsdurchsetzungsmechanismus. ${ }^{42}$ Dies gilt allerdings nur bis zu einer Untergrenze: Für Kleinstbetroffenheiten kann auch durch eine Kollektivierung kein hinreichender Anreiz hergestellt werden. ${ }^{43}$

\section{c) Anwendungspotentiale im deutschen Verwaltungsrecht}

Auch Anwendungsbereiche, in denen eine individuell geringe Massenbetroffenheit besteht, können hier nur beispielhaft benannt werden. $\mathrm{Zu}$ denken ist etwa an einen allen Anwohnern einer Hauptverkehrsstraße zustehenden Anspruch auf Aufstellung eines Luftreinhalteplans i.S.d. $\$ 47$ Abs. 1 S. 1 BImSchG bei einer geringfügigen Überschreitung von $\mathrm{NO}_{2}$-Grenzwerten. Oder an einen Rückzahlungsanspruch auf Grundlage eines öffentlich-rechtlichen Erstattungsanspruchs, soweit die einer Gebührenerhebung zugrunde liegende Satzung für ungültig erklärt wird oder die Behörde einen gesetzlichen Gebührenrahmen ermessenswidrig ausgenutzt hat.

40 Schon Kalven/Rosenfield, University of Chicago Law Review 8 (1941), 684 ff.; später Schäfer, European Journal of Law and Economics 9 (2000), 183 (184 f.); Nagy, Columbia Journal of European Law 19 (2013), 469 (473 ff.).

41 Guski, ZZP 131 (2018), 353 (372).

42 Hinzu kommt eine Präventionswirkung, weil das Realisierungsversprechen normativer Anordnungen auch bei geringer individueller Betroffenheit bewehrt wird. Die Rechtsdurchsetzungswirkung kann gegenläufig zur Gerichtsentlastung durch die prozessökonomische Wirkung wirken (auch Cassone/Ramello, European Journal of Law and Economics 32 [2011], 205 [211]).

43 Vgl. Stadler, Bündelung von Interessen im Zivilprozess, 2004, S. 8. 


\section{Systemkongruenz einer class action im deutschen Verwaltungsrechtsschutz}

Ergeben sich somit für class actions im Verwaltungsrecht Anwendungspotentiale, wirft dies Fragen nach ihrer Systemkongruenz auf. Näher zu beleuchten ist, ob und inwieweit sich class actions in das bestehende deutsche Verwaltungsrechtsschutzsystem einfügen würden. Dies wird im Folgenden aus kontrollkonzeptioneller (1.), verfassungsrechtlicher (2.) und verwaltungsprozessrechtlicher (3.) Perspektive beleuchtet.

\section{Kontrollkonzeptionelle Perspektive}

\section{a) Ausrichtung auf den Individualrechtsschutz}

Die grundrechtliche Ausgestaltung der Rechtsschutzgarantie (Art. 19 Abs. 4 GG) illustriert die gedankliche Ausrichtung des deutschen Verwaltungsrechtsschutzes auf den Individualrechtsschutz. ${ }^{44}$ Eine verwaltungsprozessuale class action tritt dieser Grundkonzeption teilweise entgegen: Einerseits implizieren class actions - anders als altruistische Verbandsklagerechte $^{45}$ - nicht, dass öffentliche Interessen ${ }^{46}$ oder eigenständige Gruppeninteressen ${ }^{47}$ verfolgt werden. Eine class action dient nicht der Eröffnung des Zugangs zu bisher einer Kontrolle verschlossenen Bereichen (nur) objektiven Rechts, sondern wahrt die Akzessorietät zwischen materieller Rechtsposition und Rechtsdurchsetzungsmacht. ${ }^{48}$

Andererseits lösen sich Gruppenklagen durchaus von der mit der Ausrichtung auf subjektive öffentliche Rechte verbundenen Engführung des deutschen Verwaltungsprozessrechts, nach der Individualrechtsschutz stets als individueller Rechtsschutz verstanden wird und auf eine kleinräumige

44 Ibler, Rechtspflegender Rechtsschutz im Verwaltungsrecht, 1999, S. 167 ff.; Marxsen, Die Verwaltung 53 (2020), 215 (218); Schmidt-Aßmann, Kohärenz und Konsistenz des Verwaltungsrechtsschutzes, 2015, S. 18.

45 Etwa im Natur- ( $\$ 64$ Abs. 1 BNatSchG) und Tierschutzrecht (etwa $\mathbb{1}$ Abs. 1 HmbTierSchVKG, $\mathbb{3}$ Abs. 1 TierSchLMVG RP); näher Schlacke, Überindividueller Rechtsschutz, 2008, S. 5 ff. m.w.N.

46 Heese, JZ 2019, 429 (430); in diese Richtung indes Bruns, NJW 2018, 2753 (2753 f.).

$47 \mathrm{Zu}$ Potentialen und Risiken einer strategischen Prozessführung, zu der auch class actions eingesetzt werden könnten, vgl. Strobel, in diesem Band, S. $157 \mathrm{ff}$.

48 Das privatnützige Vorgehen dient freilich zugleich der Bewehrung der Gesamtrechtsordnung: Gärditz, Verhandlungen des 71. Deutschen Juristentags, Bd. I, 2016, D 15, 17 f.; Mangold/Wahl, Die Verwaltung 48 (2015), 1 (17). 
Rechtsdurchsetzung begrenzt bleibt. Sie entkontextualisieren eine Rechtsstreitigkeit und eröffnen die Möglichkeit zu einer allgemein- - oder jedenfalls über die konkret Agierenden hinaus - verbindlichen Klärung einer Tatsachen- oder Rechtsfrage. ${ }^{49}$ Aus dieser Perspektive rückt die allgemeine Konfliktlösung an die Stelle punktueller Nachprüfung einer Verletzung subjektiver öffentlicher Rechte in den Mittelpunkt des Verwaltungsprozesses. ${ }^{50}$ Damit befördern class actions eine gewisse „Kolchosierung“51 des Prozesses: Sie wandeln die Rolle des Anspruchsberechtigten vom eigennutzgeleiteten Einzelkämpfer zum Mitglied eines größeren Kollektivs. In der Folge dient der Gerichtsprozess weniger als institutionalisiertes Forum für das persönliche Aufeinandertreffen der Streitenden denn als Ort zur Lösung gesellschaftlicher Konfliktlagen. Dies stärkt zugleich das gestalterischrechtserzeugende Moment gerichtlicher Entscheidungen. Ein solcher $\mathrm{Zu}$ gewinn an judikativer Entscheidungsmacht gegenüber der Exekutive kann sich, dies ist zu bedenken, ${ }^{52}$ auch auf das Kräfteverhältnis zwischen den Gewalten in der grundgesetzlichen Funktionenordnung auswirken.

\section{b) Vielschichtigkeit der Verwaltungskontrolle}

Verwaltungskontrolle zeichnet sich durch ihre Vielgestaltigkeit aus. Sie ist nicht bei den Gerichten monopolisiert, sondern wird auch durch eine politische Kontrolle, Rechnungshofkontrolle, Öffentlichkeitskontrolle, in verfassungsgebotener Achtung der Eigenständigkeit der demokratisch legitimierten Verwaltung vor allem aber durch die Verwaltung selbst ins Werk gesetzt. Diese Verfahren stehen in einem "funktionalen Zusammenhang “53 und müssen in ihren unterschiedlichen Ansatzpunkten, Wirkrichtungen und Prüfungsmaßstäben aufeinander abgestimmt werden. ${ }^{54}$ Gerade die verwaltungsinterne Kontrolle, die in Form des Widerspruchsverfahrens

49 Vgl. Bruns, NJW 2018, 2753 (2754); auch schon ders., in: Stürner/ders., Globalisierung und Sozialstaatsprinzip, 2014, S. 255 (263).

50 Entsprechend - bezogen auf den Zivilprozess - Scholz, ZG 18 (2003), 248 (261); Tolani, Parteiherrschaft und Richtermacht, 2019, S. $80 \mathrm{ff}$.

51 Stürner, ÖJZ 2014, 629 (632); zust. Karner, in: Schmidt-Kessel/Strünck/Kramme, Im Namen der Verbraucher?, 2015, S. 165 (181).

52 Zur Bedeutung der Gewaltenteilung bei der Ausgestaltung der Verwaltungskontrolle auch Schmidt-Aßmann, in: Anderheiden u.a., GS Brugger, S. 411 (419).

53 Ausf. Schmidt-Aßmann/Schenk, in: Schoch/Schneider, VwGO, Einleitung Rn. 197 (Stand: 2012).

54 Vgl. auch Kahl, in: Eifert/Möllers/Voßkuhle, GVwR, Bd. II, 3. Aufl. 2021, $₫ 45$ Rn. 255. Das bedeutet keine Identität; das „wechselseitige Abgestimmtsein“ kann 
einem gerichtlichen Rechtsschutz teilweise zwingend vorausgeht, ist derzeit regelmäßig auf ein Individualverfahren ausgerichtet. Eine verwaltungsprozessuale class action stellt das vor konzeptionelle Probleme. ${ }^{55}$ Sie führt jedenfalls zu einer stärker konfrontativen Ausrichtung und noch weiteren $^{56}$ Dominanz der gerichtlichen Verwaltungskontrolle. ${ }^{57}$ Daraus resultiert eine Konzentration auf eine nachträgliche Ergebniskontrolle nach Rechtmäßigkeitsmaßstäben, ${ }^{58}$ die hinter einer administrativen Eigenkontrolle teilweise zurückbleibt.

\section{c) Staatliche (Selbst-)Kontrolle versus "private enforcement"}

Class actions entsprechen einem individualistisch und liberal geprägten (US-amerikanischen) Staats- und Rechtsverständnis, das die private Rechtsdurchsetzung zur Verwirklichung des Gemeinwohls in ihr Zentrum stellt. ${ }^{59}$ Demgegenüber hat in Deutschland traditionell die staatliche Regulierung einen höheren Stellenwert. Dass bei Bedarf der Staat repressiv-ordnend eingreife, wird daher im Privatrecht bisweilen als Argument gegen eine Stärkung der Rechtsdurchsetzung durch kollektive Rechtsschutzinstrumente in Stellung gebracht. ${ }^{60}$ Für einen kollektiven Rechtsschutz im Verwaltungsrecht verfängt dieses Argument jedoch nur bedingt. Denn gegenüber staatlichem Handeln bestehen in Form der Staatsaufsicht teilweise nur schwache, gegenüber dem Handeln von obersten Landes- und Bundesbehörden überhaupt keine verwaltungsinternen Kontroll- und Sanktionsmechanismen. Die Möglichkeit zur Initiierung gerichtlicher Entscheidung durch eine Mobilisierung des Einzelnen ist im Verwaltungsrecht zudem besonders bedeutsam, weil die Verwaltung ihrerseits mit der Verwaltungsvollstreckung über Verfahrensmöglichkeiten verfügt, um Ansprü-

auch in einer Differenz liegen, vgl. Hoffmann-Riem, in: Schmidt-Aßmann/ders., Verwaltungskontrolle, 2001, S. 325 (361 f.).

55 Siehe noch IV.3.

56 Die bereits bestehende Gerichtszentriertheit betont $\operatorname{Kabl}$ (Fn. 54), $\$ 45$ Rn. 260.

57 Class actions können ergänzend die Öffentlichkeitskontrolle fördern, indem sie zusätzliche Aufmerksamkeit erzeugen, vgl. Effinowicz, ZJapanR 25 (2020), 95 (127).

$58 \mathrm{Zu}$ Veränderungen im Hinblick auf das „Recht als Maßstab“ der Verwaltungsgerichtsbarkeit vgl. Stepanek, in diesem Band, S. 419 (428 ff.).

59 Bruns, Globalisierung (Fn. 49), S. 255 (262); Schmidt-Aßmann, Das Verwaltungsrecht der Vereinigten Staaten von Amerika, 2021, Kap. 2 Rn. 128 ff.; ferner Poelzig, Normdurchsetzung (Fn. 27), S. 69.

60 Bruns, NJW 2018, 2753 (2754); Stürner, ÖJZ 2014, 629 (632). 
che ohne gerichtliche Prüfung durchzusetzen. Die Frage, ob eine private oder eine staatliche Rechtsdurchsetzung zweckmäßig(er) ist, stellt sich also für das Verwaltungshandeln unter anderen Vorzeichen.

\section{Verfassungsrechtliche Perspektive}

Das Verwaltungsprozessrecht ist in hohem Maße konstitutionell eingehegt und geprägt. Diesen Anforderungen muss sich auch ein kollektiver Rechtsschutz stellen.

\section{a) Dispositionsgrundsatz}

Art. 19 Abs. 4 S. 1 GG lässt sich eine Dispositionsfreiheit entnehmen, ob, wann und inwieweit Ansprüche gerichtlich geltend gemacht werden. ${ }^{61}$ In Bezug auf eine verwaltungsprozessuale class action würde dem Rechnung getragen durch ein opt-in-Modell, bei dem eine Unterwerfung unter ein Urteil nur nach aktiver Erklärung der Beteiligung erfolgt. ${ }^{62}$ Als problematischer stellt sich demgegenüber ein opt-out-Modell dar, bei dem einem Gruppenangehörigen die Beteiligung zwar ebenso anheimgestellt bleibt, er aber für eine Nicht-Beteiligung fristgebunden aktiv werden muss. Daraus resultiert die Frage, ob Art. 19 Abs. 4 S.1 GG die fremdinitiierte Durchsetzung subjektiver öffentlicher Rechte kategorisch ausschließt. ${ }^{63}$ Dagegen spricht, dass die Möglichkeit einer Fremdinitiierung nicht den individuellen Zugang zu effektivem Rechtsschutz beschränkt. Zudem kann - wie Beispiele für Klageberechtigungen Dritter zeigen ${ }^{64}$ - durchaus ein öffentliches Interesse an der Durchsetzung subjektiver öffentlicher Rechte bestehen. Die Fremdinitiierung erweist sich dann allerdings als Frage nach der Legitimationsgrundlage des zur Durchsetzung dieser Rechte berechtigten Dritten. ${ }^{65}$

61 W.-R. Schenke, in: Kahl/Waldhoff/Walter, BK-GG, Art. 19 Abs. 4 Rn. 260 (Stand: 2020); Schmidt-Aßmann, in: Maunz/Dürig, GG, Art. 19 Abs. 4 Rn. 264 (Stand: 2020).

62 Vgl. Haß, Gruppenklage (Fn. 30), S. 320.

63 Dagegen W.-R. Schenke (Fn. 61), Art. 19 Abs. 4 Rn. 260 (Stand: 2020); wohl auch Rennert, DVBl. 2015, 793 (794).

64 Vgl. etwa die Regelungen des SpruchG sowie - praktisch allerdings bedeutungslos $-\$ 8$ Abs. 4 HwO.

65 Dazu Rennert, DVBl. 2015, 793 (799f.). 
Eine Einschränkung des Dispositionsgrundsatzes liegt weiter vor, soweit für Teilnehmer der Gruppenklage die individuelle Geltendmachung prozessualer Rechte und Gestaltungsmöglichkeiten (z. B. Klagerücknahme [\$92 Abs. 1 S. 1 VwGO] oder Abschluss eines Vergleichs [ 106 S. 1 VwGO]) während des Verfahrens ausgeschlossen wird. Das Ziel der Prozessökonomie kann hier aber durchaus ein Rechtfertigungsgrund sein. ${ }^{66}$

\section{b) Anspruch auf rechtliches Gehör}

Die individualistische Prägung des Prozessrechts durch das Grundgesetz wird besonders deutlich im Anspruch auf rechtliches Gehör (Art.103 Abs. 1 GG), nach dem jedem materiell durch eine gerichtliche Entscheidung unmittelbar Betroffenen Gelegenheit zu geben ist, sich zu den tatsächlichen Gegebenheiten und zur Rechtslage zu äußern, ${ }^{67}$ und diese Äußerung vom Gericht bei der Entscheidungsfindung beachtet werden muss. ${ }^{68}$ In einem Kollektivverfahren gerät diese Kommunikationsgarantie unter Druck, soll das Ziel der Prozessökonomie nicht gefährdet werden. Zwar ist der Anspruch auf rechtliches Gehör als normgeprägtes Grundrecht durch prozessrechtliche Regelungen näher auszugestalten. ${ }^{69}$ Jedenfalls das Fehlen einer Äußerungsmöglichkeit steht aber zum verfassungsrechtlich angelegten Konnex zwischen Bindungswirkung und Beteiligungsrecht in Widerspruch. Bei einem opt-in-Modell wird insofern die selbstinitiierte und freiwillige Entscheidung für das class action-Verfahren anstelle einer ebenso möglichen individuellen Klageerhebung - auch unter Berücksichtigung des Menschenwürdebezugs des Art. 103 Abs. 1 GG $^{70}$ - teilweise als Einwilligung bzw. Grundrechtsverzicht eingeordnet. ${ }^{71}$ Bei einem opt-out-Modell, bei dem die Gewährung rechtlichen Gehörs mangels Kenntnis aller der Rechtskraft eines Urteils Unterworfenen sogar aus-

66 Ebenso Geiger, Kollektiver Rechtsschutz im Zivilprozess, 2015, S. 248.

67 Vgl. BVerfGE 103, 397 (404); 138, 64 Rn. 55; Kment, in: Jarass/Pieroth, GG, 16. Aufl. 2020, Art. 103 Rn. 11.

68 Kunig/Saliger; in: von Münch/Kunig, GG, Bd. 2, 7. Aufl. 2021, Art. 103 Rn. 18; Nolte/Aust, in: von Mangoldt/Klein/Starck, GG, Bd. III, 7. Aufl. 2018, Art. 103 Rn. $36 \mathrm{ff}$.

69 Schulze-Fielitz, in: Dreier, GG, Bd. III, 3. Aufl. 2018, Art. 103 I Rn. 27.

70 Vgl. BVerfGE 9, 89 (95); 63, 332 (337); BVerfG, NJW 2019, 41 Rn. 64.

71 So die Begründung des Gesetzentwurfs zur ZPO-Musterfeststellungsklage, BTDrs. 19/2439, S. 28; in diese Richtung ferner Heese, JZ 2019, 429 (435). Einen abstrakten Verzicht hingegen als verfassungswidrig abl. Lange, Gruppenverfahren (Fn. 8), S. 163 ff.; Röthemeyer, MDR 2019, 6 (8). 
geschlossen ist, fehlt für eine solche Einwilligung der Anknüpfungspunkt. $\mathrm{Zu}$ bedenken ist aber, dass bei einer class action einerseits einem Repräsentanten die Möglichkeit zur Beteiligung zukommt. So können Einschränkungen persönlicher Gehörswahrnehmung verfassungskonform kompensiert werden, soweit hinreichende Informationsflüsse und Einwirkungsrechte zwischen Teilnehmern der Gruppenklage und dem Repräsentanten bestehen. ${ }^{72}$ Andererseits kann in der durch Art. 19 Abs. 4 S. 1 GG mit verfassungsrechtlichem Rang ausgestatteten Garantie effektiven Rechtsschutzes ein Rechtfertigungsgrund für eine Beschränkung des vorbehaltlos gewährleisteten Gehörsanspruchs liegen. ${ }^{73}$ Soweit dem Anspruch auf rechtliches Gehör der weitere Zweck zukommt, aufgrund einer möglichst umfänglichen Sachaufklärung zu einer richtigen Entscheidung zu gelangen, ${ }^{74}$ wird dieser im Verwaltungsprozess durch eine Beschränkung in geringerem Maße gefährdet als im Zivilprozess. Denn die durch den Untersuchungsgrundsatz ( $\$ 86$ Abs. 1 S. 1 Hs. 1 VwGO) angeordnete Verpflichtung zu gerichtlich initiierter Aufklärung stellt die einer Entscheidung zugrundeliegende Tatsachenlage von dem Vorbringen der Betroffenen unabhängig(er). Der Ausfall einer Mitwirkung der rechtsschutzsuchenden Teilnehmer fällt daher im Ergebnis geringer ins Gewicht.

\section{Verwaltungsprozessrechtliche Perspektive}

Aus verwaltungsprozessrechtlicher Perspektive stellen Gruppenklagen weder die handlungsformbezogene Ausgestaltung des deutschen Verwaltungsrechtsschutzes noch die Ausrichtung auf ein kontradiktorisches Verfahren in Frage. Als problematisch erweist sich indes, dass die Zulässigkeit einer Anspruchsdurchsetzung im Wege einer class action eine gewisse Kongruenz zu den Zulässigkeitsvoraussetzungen im individuellen Rechtsschutz aufweisen muss, um diese und das mit ihnen verfolgte Ziel nicht zu unterminieren. Vor diesem Hintergrund stellen insbesondere die Fristenbindung und das Widerspruchserfordernis eine Umsetzung vor Herausforderungen. Die Übernahme der Fristenbindung der Anfechtungs- und Verpflichtungsklage ( $\$ 74$ Abs. 1 und 2 VwGO) hätte für class actions die Kon-

72 Vgl. Kment (Fn. 67), Art. 103 Rn. 48; ferner Gottwald, ZZP 91 (1978), 1 (10).

73 Auch Geiger, Rechtsschutz (Fn. 66), S. 254; Jacob, in: Gärditz, VwGO, 2. Aufl. 2018, \$93a Rn. 3; ferner BVerfGE 60, 7 (15).

74 BVerfG, Beschl. v. 15.7.2016 - 2 BvR 857/14, BeckRS 2016, 49621 Rn. 8; NJW 2019, 41 Rn. 64; Remmert, in: Maunz/Dürig, GG, Art. 103 Rn. 23 (Stand: 2016). 
sequenz, dass nur Anspruchsberechtigte zusammenfinden können, die innerhalb dieser Zeitspanne einen (Widerspruchs-)Bescheid erhalten haben. Daraus resultiert zugleich eine erhebliche Verkürzung des Zeitraums zur Organisation einer class action. Dies erforderte besondere organisatorische Vorkehrungen, etwa in Gestalt eines im Internet zugänglichen Klageregisters und einer formularunterstützten Anmeldemöglichkeit. Alternativ käme in Betracht, dass sich Anspruchsberechtigte auch nach Rechtshängigkeit noch einer class action anschließen können.

Darüber hinaus stößt eine Kollektivierung auf Umsetzungsschwierigkeiten, soweit vor Klageerhebung ein obligatorisches Widerspruchsverfahren zu durchlaufen ist ( $\$ 68$ Abs. 1 S. 1, Abs. 2 VwGO). Daraus resultieren Kosten, die gerade bei geringer individueller Betroffenheit wohl nur ein Teil der Anspruchsberechtigten zu tragen bereit ist. In der Folge kann die Überwindung der rationalen Apathie durch eine gemeinsame Klageerhebung nicht gelingen und die Rechtsdurchsetzungswirkung nicht erreicht werden. Einen Ausweg könnten bei class actions die Einführung eines

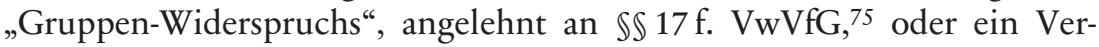
zicht auf ein Widerspruchserfordernis weisen.

\section{Rechtspolitische Erwägungen}

Aus rechtspolitischer Perspektive ist die Einführung einer class action in das deutsche Verwaltungsprozessrecht anzustreben, wenn - erstens - eine Verbesserung der Prozessökonomie und/oder die verbesserte Rechtsdurchsetzung politisch wünschenswert ist/sind und - zweitens - die Einführung für das jeweilige oder beide Ziele einen Mehrwert verspricht.

\section{Prozessökonomie}

Die Stärkung der Prozessökonomie liegt zuvörderst im Interesse an staatlicher Effizienz, dessen wirtschaftlich-finanzielle Dimension in $\$ \$ 5,6$ Abs. 1 HGrG zum Ausdruck kommt und dem bisweilen Verfassungsrang zuge-

75 Ausf. Schmel, Massenverfahren vor den Verwaltungsbehörden und Verwaltungsgerichten, 1982, S. 65 ff. 
standen wird. ${ }^{76}$ Für Bürger kann sie zu Vorteilen im Hinblick auf die Geschwindigkeit der Erlangung von Rechtsschutz führen. Einen Mehrwert für die Prozessökonomie entfaltet die Einführung einer class action aber nur, soweit nicht im Verwaltungsprozessrecht funktionale Äquivalente existieren. Bei näherer Betrachtung erweisen sich die Instrumente der VwGO im Hinblick auf ihre Eignung zu Effizienzsteigerungen bei Massenbetroffenheiten als jeweils auf spezifische Weise gegenüber einer class action beschränkt: Musterverfahren gem. $\$ 93 a$ VwGO können nur bei echter Massenbetroffenheit Anwendung finden. ${ }^{77}$ Die class action kann zudem bei identischem Streitgegenstand eine Klärung gegenüber allen Beteiligten in einer einzigen Entscheidung herbeiführen, insofern wird auch das Prozesskostenrisiko verteilt. Ebenfalls prozessökonomische Wirkung kommt der abstrakten Normenkontrolle durch ihre erga omnes-Wirkung ( $\$ 47$ Abs. 5 S. 2 VwGO) $\mathrm{zu}^{78}$ wodurch letztlich jeder an den Ausgang eines fremdinitiierten Prozesses gebunden ist. Der Rechtsschutz nach $\$ 47$ VwGO ist allerdings auf die Kontrolle untergesetzlicher Normen beschränkt und bleibt damit erheblich hinter dem im Ansatz handlungsformunabhängigen Anwendungsbereich von class actions zurück. Im Hinblick auf die Bildung einer Streitgenossenschaft ( $\$ 64 \mathrm{VwGO}$ i.V.m. $\$ \$ 59 \mathrm{ff}$. ZPO) ${ }^{79}$ sowie auf die nachträgliche Verfahrensverbindung durch das Gericht $(\$ 93 \mathrm{VwGO})^{80}$ ergeben sich für die Prozessökonomie Einbußen, weil für alle Verfahren dasselbe Gericht zuständig sein muss. Dem ließe sich bei class actions durch Festlegung einer spezifischen Zuständigkeit - in den Grenzen des Föderalismus - entgegenwirken.

76 Schmidt-Aßmann, in: Hoffmann-Riem/ders., Effizienz als Herausforderung an das Verwaltungsrecht, 1998, S. 245 (255 f.); Hyckel, GVRZ 2020, 5 knüpft ein Effizienzgebot an das Demokratieprinzip (Rn. 26) und Art. 114 Abs. 2 S. 1 GG (Rn. 28).

77 Vgl. Garloff, in: Posser/Wolff, VwGO, 2. Aufl. 2014, \$93a Rn. 1. Eine Erweiterung auf unechte Massenverfahren erwägend Völzmann, in: Greve u.a., 60. Assistententagung Öffentliches Recht, 2020, S. 289 (302 ff.); für eine Gesetzesänderung plädiert Beckermann, GVRZ 2019, 16 Rn. 56. Diskutiert wird die Anwendbarkeit auf unechte Massenverfahren ferner für $\$ 114$ a SGG: befürwortend Kummer, in: Peters/Sautter/Wolff, Kommentar zur Sozialgerichtsbarkeit, $\mathbb{1} 114 \mathrm{a}$ Rn. 7 (Stand: 2009); abl. Roller, in: Berchtold, SGG, 6. Aufl. 2021, $\$ 114$ a Rn. 4.

78 Ehlers, in: ders./Schoch, Rechtsschutz im Öffentlichen Recht, 2009, $\$ 47$ Rn. 1; Hyckel, Prozessökonomie (Fn. 21), S. 422.

79 Vgl. Czybulka/Siegel, in: Sodan/Ziekow, VwGO, 5. Aufl. 2018, \$ 64 Rn. 33; Krausnick, in: Gärditz, VwGO, 2. Aufl. 2018, $₫ 64$ Rn. 5. Im Hinblick auf die Einführung zivilprozessualer Gruppenklagen Hopt/Baetge, in: Basedow/Hopt/Kötz/Baetge, Die Bündelung gleichgerichteter Interessen im Prozess, 1999, S. 11 (54).

80 Vgl. Peters/Pätzold, in: Sodan/Ziekow, VwGO, 5. Aufl. 2018, $\mathbb{9 3}$ Rn. 4; Rudisile, in: Schoch/Schneider, VwGO, $\mathbb{9} 93$ Rn. 6 (Stand: 2009). 
In der Praxis werden insbesondere im Hochschulzulassungsrecht sog. Sammelbeschlüsse bzw. -urteile erlassen. Die Ausgangsverfahren bleiben dabei prozessrechtlich eigenständig, gleichwohl ergeht eine Entscheidung mit einheitlicher Begründung. ${ }^{81}$ Diese Verfahrensweise kommt im Ergebnis einer class action nahe, ist aber schon deshalb nachteilig, weil sie gesetzlich ungeregelt bleibt und für prozessökonomische Vorteile wie die spezifische Ausgestaltung des rechtlichen Gehörs keinen Anknüpfungspunkt bietet.

\section{Rechtsdurchsetzung}

Aus rechtspolitischer Warte stellt sich weiter die Frage, ob und inwieweit die möglichst umfängliche Rechtsdurchsetzung gemeinwohlförderlich ist. Im privatrechtlichen Kontext besteht Bedarf für die Internalisierung auch von Bagatellschäden, um ein optimales Sorgfaltsniveau zu erreichen ${ }^{82}$ und Fehlallokationen mit der Folge gesamtgesellschaftlicher Wohlfahrtsverlus$\mathrm{te}^{83} \mathrm{zu}$ vermeiden. Auf das Verwaltungsrecht lässt sich dies angesichts einer rechtsgebundenen und grundsätzlich nicht profitorientiert agierenden Verwaltung nicht übertragen. Auch ein legitimatorischer Mehrwert im Hinblick auf Art. 20 Abs. 2 GG lässt sich der gerichtlichen Kontrolle nicht ohne weiteres zuschreiben, verhilft sie doch demokratisch gesetztem Recht bloß zur Durchsetzung. ${ }^{84}$ Demokratisch relevant wird gerichtliche Kontrolle allenfalls durch die einem Gerichtsverfahren innewohnende Mitwirkungsmöglichkeit und Befriedungsfunktion ${ }^{85}$. Eine möglichst effektive gerichtliche Kontrolle lässt sich aber als Ausdruck des verfassungsrechtlichen Rechtsstaatsprinzips (Art. 20 Abs. 3, 28 Abs. 1 S. 1 GG) begreifen. ${ }^{86}$ Schon die Existenz von Gerichtskosten zeigt - wie verschiedene andere Regelungen ${ }^{87}$ - jedoch, dass sich der Gesetzgeber bislang gegen eine möglichst vollumfängliche Rechtsdurchsetzung verwahrt. Dahinter steht die Einsicht, dass gerichtlicher Rechtsschutz „eine wichtige, aber keine un-

81 Vgl. nur OVG MV, NVwZ-RR 1994, 334 (334); NJOZ 2005, 1357 (1357).

82 Haß, Gruppenklage (Fn. 30), S. 50 f.; Schwalbe, Verbraucher (Fn. 31), S. 23 (27).

83 Vgl. Karner, Verbraucher (Fn. 51), S. 165 (172).

84 Auch Grzeszick, in: Maunz/Dürig, GG, Art. 20 II Rn. 168 (Stand: 2010) m.w.N.; anders indes BVerfGE 151, 202 Rn. 130; Huber, DVBl. 2021, 753 (760).

85 Dazu Lubmann, Legitimation (Fn. 29), S. 82 ff.

86 Vgl. Kahl (Fn. 54), \$ 45 Rn. 73.

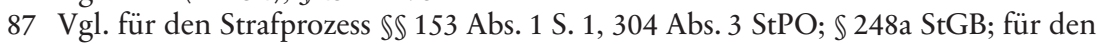
Zivilprozess $₫ 511$ Abs. 2 Nr. 1 ZPO. 
erschöpflich verfügbare Ressource“ ${ }^{88}$ ist. Der Zugang zu gerichtlicher Rechtsdurchsetzung betrifft daher auch unmittelbar die Allokation staatlicher Ressourcen. ${ }^{89}$

Der rechtspolitische Blick ist weiter - neben Alternativen wie der Einführung zusätzlicher Verbandsklagerechte oder einer Änderung des Gerichtskostenrechts - auf bestehende funktionale Äquivalente zu richten. Im Verwaltungsrecht kommt schon der Möglichkeit der Erhebung eines Widerspruchs gewisse Rechtsdurchsetzungswirkung $\mathrm{zu}$, ist diese doch grundsätzlich mit niedrigeren Kosten verbunden als die Klageerhebung. ${ }^{90}$ Gleichwohl verbleiben auch hier individuelle Kosten, die einer Rechtsdurchsetzung bisweilen entgegenstehen. ${ }^{91}$ Zudem kann die Rechtsdurchsetzungswirkung offenkundig nicht erzielt werden, soweit das Widerspruchsverfahren gem. $\mathbb{} 68$ Abs. 1 S. 2 VwGO (ggf. i.V.m. landesgesetzlicher Regelung) unstatthaft ist oder gegenüber dem Betroffenen kein Verwaltungsakt ergangen ist. ${ }^{92}$ Im gerichtlichen Verfahren kann die Bildung einer Streitgenossenschaft die psychologische Hemmschwelle für ein gerichtliches Vorgehen reduzieren. Sie hat allerdings nur geringen Einfluss auf den für einen Prozess zu betreibenden Aufwand, daher wird sie die Rationalität der Apathie nur selten überwinden können. ${ }^{93}$

\section{Ausgestaltung}

Der Gesetzgeber kann durch die genauere Ausgestaltung den beiden zentralen Wirkungen - Rechtsdurchsetzung und Prozessökonomie - in unterschiedlichem Maße Wirkkraft verleihen. Grundsätzlich wäre eine Entscheidung zwischen einem opt-in- und einem opt-out-Modell zu treffen. Klärungsbedürftig wäre ferner, ob auch Teilaspekte kollektivierungsfähig sind und wo die erstinstanzliche Zuständigkeit anzusiedeln wäre. Schließlich müsste entschieden werden, wem die Repräsentationsbefugnis zukommt, und das Kostenrecht entsprechend ausgestaltet werden, um hinreichende Klageanreize zu setzen. Zur eingehenden Erörterung dieser und weiterer

88 Schmidt-Aßmann, Verwaltungsrechtliche Dogmatik, 2013, S. 108.

89 Buchheim, Actio, Anspruch, subjektives Recht, 2017, S. 95; Gärditz, Verhandlungen (Fn. 48), D 47.

$90 \mathrm{Zu}$ den rechtlichen Regelungen Poschenrieder, Außergerichtliches Vorverfahren im Verwaltungsrecht, 2019, S. $226 \mathrm{ff}$.

91 Siehe auch oben IV. 3.

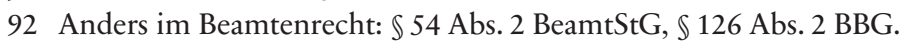

93 Auch - für das Zivilprozessrecht - Meller-Hannich, Verhandlungen (Fn. 17), A 57. 
Fragen der Ausgestaltung fehlt hier der notwendige Raum, sie muss daher anderer Stelle vorbehalten bleiben.

\section{Fazit}

Die Auseinandersetzung mit class actions führt die Steuerungswirkungen des vermeintlich technischen Prozessrechts vor Augen. Bei näherer Betrachtung sind class actions gewiss kein Allheilmittel zur Sicherstellung eines zukunftsfähigen Verwaltungsrechtsschutzes. Bei entsprechender Ausgestaltung könnten sich aber die beiden zentralen Wirkungen - ein prozessökonomischer Rechtsschutz bei individuell mittlerer und starker Massenbetroffenheit und eine verbesserte Rechtsdurchsetzung bei individuell geringer Massenbetroffenheit - auch im Verwaltungsprozessrecht erreichen lassen. Eine solche Veränderung wäre kein minimalinvasiver Eingriff, sondern eine grundlegende Innovation. Insbesondere aus der Fristenbindung und dem Erfordernis eines erfolglosen Widerspruchs ergeben sich für die Ausgestaltung Schwierigkeiten. Gelingt es, diese zu überwinden, haben class actions als Sinnbild eines „kollektiven Individualrechtsschutzes" zumindest bedenkenswerte Potentiale für den Zugang zum Verwaltungsrecht. 
